\title{
CAPACIDAD DIAGNÓSTICA DEL GDx VCC PARA EL DIAGNÓSTICO DEL GLAUCOMA
}

\section{DIAGNOSTIC ABILITY OF GDx VCC FOR GLAUCOMA DIAGNOSIS}

\author{
BORQUE E ${ }^{1}$, FERRERAS A ${ }^{2}$, POLO V V ${ }^{2}$ LARROSA JM ${ }^{2}$, PABLO LE ${ }^{2}$, HONRUBIA FM $^{2}$
}

\section{RESUMEN}

Objetivo: Evaluar la capacidad diagnóstica de los distintos parámetros que ofrece el GDx VCC para discriminar entre ojos sanos y glaucomatosos.

Material y métodos: Se seleccionaron de forma prospectiva y consecutiva 417 ojos de 417 pacientes. Fueron divididos en 60 controles, 218 hipertensos oculares, 68 glaucomas preperimétricos y 71 glaucomas según la presión intraocular, la morfología papilar y los resultados en la perimetría automatizada convencional A todos ellos se les realizó una polarimetría láser con el GDx VCC. Se compararon los parámetros de la capa de fibras nerviosas de la retina (CFNR) del GDx-VCC entre los grupos. Se calcularon las curvas ROC entre el grupo normal y de glaucomas. Se consideraron como mejores parámetros aquellos con mejor área bajo la curva ROC y el mejor balance de sensibilidad/especificidad.

Resultados: La mayoría de los parámetros del GDx-VCC mostraron diferencias entre el grupo de glaucomas y el resto de los grupos. Algunos parámetros fueron también diferentes al comparar el grupo normal y el de glaucomas preperimétricos. El mejor de ellos fue el indicador de fibras nerviosas (NFI; área bajo la curva ROC: 0,876). El NFI, el área inferior normalizada y el área superior norma-

\section{ABSTRACT}

Purpose: To assess the diagnostic ability of scanning laser polarimetry (GDx VCC) to differentiate between normal and glaucomatous eyes.

Methods: 417 eyes of 417 consecutive subjects were prospectively selected. They were classified into 60 healthy controls, 218 ocular hypertensive eyes, 68 glaucoma suspects, and 71 glaucomatous eyes, depending on intraocular pressure, optic nerve head morphology and standard automated perimetry results. All underwent a scanning laser polarimetry with the GDx VCC. Retinal nerve fiber layer (RNFL) parameters of the GDx-VCC were compared among the four groups. Receiver operating characteristic (ROC) curves were plotted between normal and glaucomatous eyes. The best parameters were defined according to the area under the ROC curve and the best sensitivity/specificity balance.

Results: Most parameters of GDx-VCC exhibited differences between the glaucoma group and the rest of the groups. Some parameters were also different between healthy patients and glaucoma suspects. The best parameter was the nerve fiber indicator (NFI; area under the ROC curve: 0.876). NFI, superior normalized area, and inferior normalized area yielded the highest sensitivities at $85 \%$ and 90\% fixed specificity.

\footnotetext{
Recibido: 3/9/07. Aceptado: 14/5/08.

Servicio de Oftalmología. Hospital Universitario Miguel Servet. Instituto Aragonés de Ciencias de la Salud. Zaragoza. España.

1 Licenciado en Medicina.

2 Doctor en Medicina.

Correspondencia:

Enrique Borque Rodríguez-Maimón

Consultas Oftalmología Hospital Universitario Miguel Servet

Isabel la Católica, 1-3

50009 Zaragoza

España

E-mail: eborque@gmail.com
} 
lizada presentaron las sensibilidades más altas para especificidades fijadas en el $85 \%$ y $90 \%$.

Conclusiones: La mayoría de los parámetros de la CFNR medidos con el GDx-VCC ofrecieron una buena capacidad para el diagnóstico del glaucoma de ángulo abierto. El mejor parámetro del GDxVCC para diferenciar entre normales y glaucomas fue el NFI.

Palabras clave: Polarimetría láser, GDx VCC, glaucoma, diagnóstico.
Conclusions: Most RNFL parameters measured with the GDx-VCC provided good diagnostic ability for open-angle glaucoma. The best GDx-VCC parameter in differentiating between normal and glaucomatous eyes was the NFI (Arch Soc Esp Oftalmol 2008; 83: 357-364).

Key words: Laser polarimetry, GDx VCC, glaucoma, diagnosis.

\section{INTRODUCCIÓN}

El glaucoma primario de ángulo abierto es una neuropatía óptica multifactorial en la que se produce una pérdida progresiva de los axones de las células ganglionares. El daño en la capa de fibras nerviosas de la retina (CFNR) se sigue habitualmente de una serie de cambios estructurales en la cabeza del nervio óptico y de defectos característicos en el campo visual $(1,2)$.

Algunos estudios han sugerido que las alteraciones en la CFNR pueden detectarse hasta 6 años antes de la aparición de defectos consistentes en el campo visual (3). Con el fin de superar la subjetividad en la valoración de los métodos tradicionales de evaluación de la CFNR, como las fotografías monocromáticas $(4,5)$, en los últimos años han aparecido diversos equipos capaces de obtener imágenes y datos cuantitativos de la CFNR de forma objetiva y reproducible. Una de estas tecnologías es la polarimetría láser (GDx) cuya última versión comercializada incluye un compensador corneal variable (VCC) de la birrefringencia del polo anterior (6-8). El GDx-VCC estima el espesor de la CFNR midiendo el retardo en el haz de un láser polarizado, inducido por los microtúbulos birrefringentes de los axones de las células ganglionares $(9,10)$. El GDx-VCC analiza una franja de la CFNR peripapilar y ofrece al clínico diversos mapas y parámetros, tanto globales como relativos, e incluye los de mayor importancia en el informe resumido que presenta. En este informe también se incluye el indicador de fibras nerviosas (NFI) y los parámetros relativos a la curva que representa el espesor de la capa de fibras nerviosas, curva TSNIT (promedio TSNIT, promedio superior, promedio inferior, y desviación estándar TSNIT), que se basan en un cálculo que el aparato establece de forma automáti- ca en un círculo alrededor de la papila. El NFI es un parámetro global basado en el mapa de espesor que se calcula a partir de redes neuronales denominado Support Vector Machine (SVM). Los resultados de los parámetros TSNIT se comparan con una base de datos normalizada que el aparato incorpora en función de la edad y grupo étnico, para ser estratificados por niveles de probabilidad.

Diversos factores pueden modificar la validez interna y externa de un estudio de precisión diagnóstica. Debido a ello, se desarrolló la iniciativa STARD (Standards for Reporting of Diagnostic Accuracy) (11). El propósito de la iniciativa STARD es mejorar la calidad de los estudios de capacidad diagnóstica. Por ello, el diseño de este estudio se realizó de acuerdo a los 25 puntos definidos en la iniciativa STARD.

El objetivo del presente estudio es evaluar la capacidad diagnóstica de los distintos parámetros que ofrece el GDx-VCC para discriminar entre ojos sanos, hipertensos oculares (HTO), glaucomas preperimétricos y glaucomatosos.

\section{SUJETOS, MATERIAL Y MÉTODOS}

El diseño del estudio fue aceptado por el comité de ética del hospital, todos los participantes firmaron el consentimiento informado y la metodología del estudio se realizó siguiendo las directrices de la Declaración del Helsinki (1964).

\section{Sujetos}

De enero de 2006 a diciembre del 2006 se preseleccionaron un total de 440 sujetos de forma prospectiva y consecutiva a partir de las consultas exter- 
nas del servicio de oftalmología del Hospital Universitario Miguel Servet de Zaragoza. Todos los pacientes habían sido remitidos desde los centros de especialidades dependientes de nuestro Hospital con el objetivo de realizar un diagnóstico precoz de la enfermedad en sujetos sospechosos o para el seguimiento o tratamiento de sujetos glaucomatosos. En 11 de ellos fue imposible obtener al menos una de las pruebas fiables o de calidad suficiente, cinco no firmaron el consentimiento informado, y siete no acudieron a todas las citas para completar el protocolo exploratorio. Finalmente, se incluyeron 417 sujetos de raza caucásica. Se consideró para el estudio un ojo de cada sujeto. La elección se hizo de forma aleatoria, salvo que sólo uno de los dos ojos cumpliera los criterios de inclusión.

Todos los sujetos, con independencia de su grupo de clasificación, cumplieron unos criterios de inclusión que fueron: edad entre 18 y 80 años, agudeza visual superior a 20/30 en la escala de Snellen, defecto refractivo inferior a 5 dioptrías de esfera y de 3 dioptrías de cilindro, medios ópticos transparentes (color u opalescencia nuclear, cortical o subcapsular posterior del cristalino $<1$ según la Lens Opacities Classification System III) (12) y aceptación del consentimiento informado. Se excluyeron aquellos individuos con antecedentes de cirugía o traumatismos oculares graves, enfermedades sistémicas de repercusión oftálmica, o imposibilidad de realizar o valorar cualquiera de las pruebas incluidas en el protocolo exploratorio.

\section{Protocolo exploratorio}

A todos los sujetos se les realizó una exploración oftalmológica completa, que incluyó biomicroscopía con lámpara de hendidura, gonioscopía, medición de los niveles de presión intraocular (PIO) basal mediante tonometría de aplanación de Goldmann (media de tres tomas en días diferentes sin tratamiento hipotensor), paquimetría corneal central (DGH Technology, modelo DGH 500, Exton, PA, USA), evaluación del fondo de ojo mediante oftalmoscopía indirecta y en lámpara de hendidura (con lente Volk de 78 dioptrías), estereofotografías de la papila, perimetría automatizada convencional (PA) y una polarimetría láser con el GDx-VCC.

La evaluación clínica del nervio óptico fue realizada con estereofotografias papilares por dos especialistas en glaucoma enmascarados con respecto a la historia clínica del sujeto. Las discrepancias entre observadores fueron resueltas por consenso. Se definió la apariencia del nervio óptico compatible con glaucoma como la existencia de un adelgazamiento del anillo neurorretiniano, focal o difuso con aumento de la excavación o ambas (13).

La PA se realizó con el Analizador de Campo «Humphrey 750» (Zeiss-Humphrey, Dublin, CA, EEUU) (estrategia 24-2 SITA Standard). Los defectos perimétricos fueron definidos por la presencia de un grupo de al menos tres puntos alterados con un nivel de probabilidad menor a $5 \%$ o un grupo de al menos dos puntos alterados con un nivel de probabilidad menor a $1 \%$ (se excluyeron los puntos situados en los polos de la mancha ciega) (14) y/o desviación estándar de la media (DSM) con un nivel de probabilidad menor al 5\%; y/o prueba del hemicampo glaucomatoso fuera de límites normales. Se realizaron al menos dos PAs para disminuir el efecto aprendizaje, y si alguna de ellas no cumplió los criterios de validez definidos por el propio perímetro (falsos positivos, falsos negativos y pérdida de fijación), la prueba fue repetida.

Las medidas de la CFNR peripapilar se realizaron con el polarímetro láser GDx-VCC (Laser Diagnostic Technologies, Inc., San Diego, CA, EEUU). El examen se realizó bajo midriasis por dos técnicos experimentados. En un primer paso el aparato compensó la birrefringencia del segmento anterior con el método descrito por Zhou y Weinreb (15), basándose en el patrón de retardo de la región macular (magnitud y eje). Todos los exámenes fueron de alta calidad (mayor de 7) centrados en el nervio óptico, con imágenes perfectamente enfocadas e iluminadas de manera uniforme sin artefactos por movimiento. En caso de no cumplir los requisitos de calidad la prueba fue repetida.

El software del GDx-VCC (versión 5.4.1.35) basándose en los valores de retardo dentro del círculo de cálculo peripapilar ofrece los parámetros TSNIT. Otros parámetros analizados en nuestro estudio fueron: NFI, radio superior, radio inferior, superior/nasal, modulación máxima, máximo superior, máximo inferior, modulación de la elipse, área normalizada superior, área normalizada inferior, temporal/nasal, inferior/nasal, integral total, integral superior, inferior, promedio temporal y promedio nasal. El percentil 95 fue usado como punto de corte para clasificar la CFNR como dentro de límites normales (valor de 0 a 30) o en la frontera (valores de 31-50). El 99\% de confianza fue usado como 
punto de corte para valores fuera de límites normales (51-100).

\section{Clasificación en grupos}

Los sujetos incluidos fueron clasificados en cuatro grupos diagnósticos: normal o control, HTO, glaucomas preperimétricos y glaucoma.

- Grupo control (60 ojos): Exploración oftalmológica normal, PIO menor de $21 \mathrm{mmHg}$, morfología del nervio óptico normal y PA normal.

- Grupo de HTO (218 ojos): PIO mayor o igual a $21 \mathrm{mmHg}$, morfología papilar normal y PA normal.

- Grupo de glaucoma preperimétrico (68 ojos): PIO mayor o igual a $21 \mathrm{mmHg}$, morfología papilar compatible con glaucoma y PA normal.

- Grupo de glaucomas (71 ojos): nervio óptico compatible con glaucoma y PA alterada.

\section{Análisis estadístico}

Para realizar los cálculos y análisis estadísticos se utilizaron los programas estadísticos SPSS (versión 15.0; SPSS Inc., Chicago, IL, EEUU) y MedCalc (versión 9.3.2.0 MedCalc Software, Belgium).

Se realizó un análisis de la varianza (ANOVA) de los parámetros del GDx-VCC entre los cuatro grupos diagnósticos con la corrección de Bonferroni para múltiples comparaciones.

Entre los grupos de sujetos normales y glaucomatosos se calcularon las curvas ROC (receiver operating characteristic) para cada uno de los parámetros de la CFNR. La curva ROC es una representación de la tasa de falsos positivos (1-especificidad) frente a la tasa de verdaderos positivos (sensibilidad). Es una manera útil de mostrar la relación continua entre sensibilidad y especificidad de una prueba o medida. Cuanto más se acerque la curva ROC a la esquina superior izquierda de la gráfica, mejor rendimiento diagnóstico presentará la prueba.

El área bajo la curva ROC (ABC) es un valor numérico que nos da una medida de la capacidad diagnóstica de un test. Una prueba perfecta tendría un $\mathrm{ABC}$ de 1 (100\% de sensibilidad y $100 \%$ de especificidad), mientras que un test sin ningún valor diagnóstico tendría un $\mathrm{ABC}$ de 0,5. Para establecer si hubo diferencias en las ABC se utilizó el método descrito por Hanley McNeil (16).

También se calcularon los valores de sensibilidad de los parámetros del GDx-VCC para especificidades fijadas en el $85 \%$ y $90 \%$, para discriminar entre ojos normales y glaucomatosos.

\section{RESULTADOS}

En la tabla I se incluyen las características demográficas de los grupos del estudio. La edad de los hipertensos oculares fue menor que la del resto de los grupos, y la de los glaucomatosos mayor, siendo similares e intermedias la edad de los normales y glaucomas preperimétricos. Los glaucomas presentaron una mejor agudeza visual corregida menor que los otros grupos. La PIO, relación excavación/disco vertical (valorada en estereofotografías) y los valores de los índices perimétricos fueron diferentes entre los grupos según los criterios de clasificación definidos en el apartado de métodos. Los hipertensos oculares presentaron córneas más gruesas que el resto de los grupos.

Tabla I. Características clínicas de la población del estudio

\begin{tabular}{|c|c|c|c|c|c|c|c|c|c|c|c|c|}
\hline & \multicolumn{3}{|c|}{ Normal $(n=60)$} & \multicolumn{3}{|c|}{ HTO $(n=218)$} & \multicolumn{3}{|c|}{ Glaucoma preperimétrico $(\mathrm{n}=68)$} & \multicolumn{3}{|c|}{ Glaucoma $(\mathrm{n}=71)$} \\
\hline & Media & $\mathrm{DE}$ & $\mathrm{p}$ & Media & $\mathrm{DE}$ & $\mathrm{p}$ & Media & $\mathrm{DE}$ & $\mathrm{p}$ & Media & $\mathrm{DE}$ & $\mathrm{p}$ \\
\hline Edad & 59,85 & 10,78 & $\mathrm{H}$ & 53,21 & 12,01 & $\mathrm{~N}, \mathrm{P}, \mathrm{G}$ & 59,57 & 10,18 & $\mathrm{H}$ & 64,83 & 9,23 & $\mathrm{H}$ \\
\hline MAVC & 0,89 & 0,10 & G & 0,93 & 0,10 & G & 0,89 & 0,12 & G & 0,83 & 0,13 & $\mathrm{~N}, \mathrm{H}, \mathrm{P}$ \\
\hline PIO Basal & 14,39 & 2,45 & $\mathrm{H}, \mathrm{P}, \mathrm{G}$ & 23,15 & 2,92 & $\mathrm{~N}, \mathrm{G}$ & 22,51 & 3,44 & $\mathrm{~N}, \mathrm{G}$ & 24,14 & 3,85 & $\mathrm{~N}, \mathrm{H}, \mathrm{P}$ \\
\hline $\mathrm{E} / \mathrm{D}$ & 0,29 & 0,17 & $H, P, G$ & 0,41 & 0,13 & $\mathrm{~N}, \mathrm{P}, \mathrm{G}$ & 0,69 & 0,16 & $\mathrm{~N}, \mathrm{H}$ & 0,72 & 0,19 & $\mathrm{~N}, \mathrm{H}$ \\
\hline DM PA & $-1,35$ & 2,41 & G & $-0,26$ & 1,06 & G & $-0,43$ & 1,30 & G & $-6,10$ & 5,43 & $\mathrm{~N}, \mathrm{H}, \mathrm{P}$ \\
\hline DSM PA & 1,51 & 1,61 & G & 0,96 & 0,76 & G & 0,97 & 0,72 & G & 5,03 & 3,62 & $\mathrm{~N}, \mathrm{H}, \mathrm{P}$ \\
\hline Paquimetría & 551,5 & 20,24 & $\mathrm{H}$ & 572,6 & 27,38 & $\mathrm{~N}, \mathrm{P}, \mathrm{G}$ & 550,9 & 22,41 & $\mathrm{H}$ & 543,3 & 26,54 & $\mathrm{H}$ \\
\hline
\end{tabular}

HTO = hipertensos oculares; DE = desviación estándar; $\mathrm{p}=$ diferencias significativas con; MAVC = mejor agudeza visual corregida; PIO = presión intraocular; $\mathrm{E} / \mathrm{D}=$ relación excavación /disco vertical valorada en estereofotografías; $\mathrm{DM}=$ desviación media; $\mathrm{PA}=$ perimetría automatizada convencional; DSM = desviación estándar de la media. N: Diferencias con el grupo normal; H: Diferencias con el grupo de HTO; P: Diferencias con el grupo de glaucomas preperimétricos; G: Diferencias con el grupo de glaucomas. 
Todos los parámetros estudiados mostraron diferencias entre los glaucomas y los demás grupos (tabla II), excepto el cociente superior/nasal (no mostró diferencias entre los glaucomas y los normales), la modulación de la elipse (no se encontraron diferencias entre los glaucomas y los glaucomas preperimétricos), el cociente temporal/nasal (no hubo diferencias entre los glaucomas y los glaucomas preperimétricos), el promedio temporal (no se observaron diferencias entre los glaucomas y los normales y glaucomas preperimétricos), y el promedio nasal (no presentó diferencias entre los glaucomas y los glaucomas preperimétricos). Ningún parámetro mostró diferencias entre controles e hipertensos oculares.

La tabla III muestra las ABCs de todos los parámetros del GDx-VCC para discriminar entre controles y glaucomatosos. El parámetro con mayor ABC fue el NFI (0,876). Le siguen el área inferior normalizada $(0,847)$ y el promedio inferior $(0,822)$. Las curvas ROC de los seis mejores parámetros pueden verse en la figura 1. Al comparar las curvas ROC de estos seis parámetros, el NFI presentó un

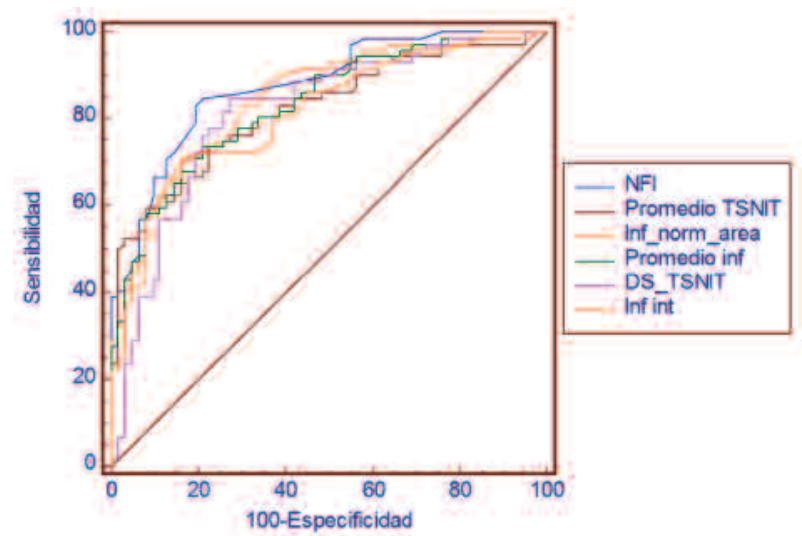

Fig. 1: Curvas ROC de los 6 parámetros del GDx-VCC con mayor capacidad diagnóstica para diferenciar entre normales y glaucomas.

$N F I=$ indicador de fibras nerviosas; Inf Norm Area = Área inferior normalizada; Promedio Inf = Promedio inferior; Desv St TSNIT = Desviación estándar TSNIT; Inf Int = Integral inferior .

ABC mayor que la del promedio TSNIT $(\mathrm{p}=0,012)$ y que la de la integral inferior $(\mathrm{p}=0,009)$.

Tabla II. Media y desviación estándar de los parámetros del GDx-VCC estudiados en cada grupo. La columna p expresa los resultados de las comparaciones múltiples (ANOVA) entre los grupos

\begin{tabular}{|c|c|c|c|c|c|c|c|c|c|c|c|c|}
\hline & \multicolumn{2}{|c|}{ Normal } & \multirow[b]{2}{*}{$\mathrm{p}$} & \multicolumn{3}{|c|}{ HTO } & \multicolumn{3}{|c|}{ Glaucoma preperimétrico } & \multicolumn{3}{|c|}{ Glaucoma } \\
\hline & Media & $\mathrm{DE}$ & & Media & $\mathrm{DE}$ & $\mathrm{p}$ & Media & DE & $\mathrm{p}$ & Media & $\mathrm{DE}$ & $\mathrm{p}$ \\
\hline NFI & 15,68 & 9,18 & $\mathrm{P}, \mathrm{G}$ & 17,64 & 7,68 & $\mathrm{P}, \mathrm{G}$ & 23,35 & 11,00 & $\mathrm{~N}, \mathrm{H}, \mathrm{G}$ & 40,90 & 21,93 & $\mathrm{~N}, \mathrm{H}, \mathrm{P}$ \\
\hline Promedio TSNIT & 56,63 & 6,35 & $\mathrm{P}, \mathrm{G}$ & 55,02 & 4,74 & G & 53,09 & 6,33 & $\mathrm{~N}, \mathrm{G}$ & 46,82 & 8,46 & $\mathrm{~N}, \mathrm{H}, \mathrm{P}$ \\
\hline Promedio superior & 66,91 & 8,69 & $\mathrm{G}$ & 66,66 & 6,96 & $\mathrm{P}, \mathrm{G}$ & 63,21 & 8,12 & $\mathrm{H}, \mathrm{G}$ & 54,73 & 11,60 & $\mathrm{~N}, \mathrm{H}, \mathrm{P}$ \\
\hline Promedio inferior & 66,48 & 8,78 & $P, G$ & 64,24 & 7,27 & G & 61,44 & 9,45 & $\mathrm{~N}, \mathrm{G}$ & 52,07 & 11,62 & $\mathrm{~N}, \mathrm{H}, \mathrm{P}$ \\
\hline Desv.est. TSNIT & 22,63 & 4,92 & G & 22,91 & 5,04 & $\mathrm{P}, \mathrm{G}$ & 20,33 & 5,38 & $\mathrm{H}, \mathrm{G}$ & 16,32 & 4,73 & $\mathrm{~N}, \mathrm{H}, \mathrm{P}$ \\
\hline Razón superior & 3,21 & 1,25 & G & 3,44 & 1,25 & G & 3,00 & 1,13 & G & 2,26 & 0,89 & $\mathrm{~N}, \mathrm{H}, \mathrm{P}$ \\
\hline Razón inferior & 3,34 & 1,23 & G & 3,45 & 1,21 & G & 3,03 & 1,17 & G & 2,31 & 0,83 & $\mathrm{~N}, \mathrm{H}, \mathrm{P}$ \\
\hline Sup/nasal & 2,31 & 0,58 & & 2,54 & 0,74 & G & 2,44 & 0,75 & G & 2,02 & 0,50 & $\mathrm{H}, \mathrm{P}$ \\
\hline Max mod & 2,58 & 1,16 & G & 2,68 & 1,22 & G & 2,26 & 1,15 & G & 1,63 & 0,79 & $\mathrm{~N}, \mathrm{H}, \mathrm{P}$ \\
\hline Máximo superior & 78,35 & 12,41 & G & 78,29 & 10,52 & G & 76,51 & 11,72 & G & 64,33 & 13,90 & $\mathrm{~N}, \mathrm{H}, \mathrm{P}$ \\
\hline Máximo inferior & 82,30 & 11,61 & G & 78,87 & 10,51 & G & 77,79 & 13,74 & G & 66,82 & 14,63 & $\mathrm{~N}, \mathrm{H}, \mathrm{P}$ \\
\hline Ell mod & 4,07 & 1,77 & $\mathrm{G}$ & 4,14 & 1,75 & G & 3,54 & 1,80 & & 2,80 & 1,30 & $\mathrm{~N}, \mathrm{H}$ \\
\hline Área sup norm & 0,14 & 0,03 & $\mathrm{P}, \mathrm{G}$ & 0,14 & 0,02 & $P, G$ & 0,12 & 0,03 & $\mathrm{~N}, \mathrm{H}, \mathrm{G}$ & 0,10 & 0,03 & $\mathrm{~N}, \mathrm{H}, \mathrm{P}$ \\
\hline Área inf norm & 0,15 & 0,03 & $P, G$ & 0,14 & 0,02 & $P, G$ & 0,13 & 0,03 & $\mathrm{~N}, \mathrm{H}, \mathrm{G}$ & 0,10 & 0,03 & $\mathrm{~N}, \mathrm{H}, \mathrm{P}$ \\
\hline Temp/nasal & 0,80 & 0,32 & G & 0,79 & 0,26 & G & 0,85 & 0,21 & & 0,97 & 0,28 & $\mathrm{~N}, \mathrm{H}$ \\
\hline Inf/nasal & 2,42 & 0,59 & $\mathrm{G}$ & 2,55 & 0,71 & G & 2,45 & 0,73 & G & 2,09 & 0,46 & $\mathrm{~N}, \mathrm{H}, \mathrm{P}$ \\
\hline Integral total & 0,53 & 0,06 & $P, G$ & 0,52 & 0,04 & G & 0,50 & 0,06 & $\mathrm{~N}, \mathrm{G}$ & 0,45 & 0,08 & $\mathrm{~N}, \mathrm{H}, \mathrm{P}$ \\
\hline Integral superior & 0,20 & 0,03 & $P, G$ & 0,20 & 0,02 & $P, G$ & 0,19 & 0,03 & $\mathrm{~N}, \mathrm{H}, \mathrm{G}$ & 0,17 & 0,03 & $\mathrm{~N}, \mathrm{H}, \mathrm{P}$ \\
\hline Integral inferior & 0,21 & 0,03 & $\mathrm{P}, \mathrm{G}$ & 0,20 & 0,02 & G & 0,19 & 0,03 & $\mathrm{~N}, \mathrm{G}$ & 0,17 & 0,03 & $\mathrm{~N}, \mathrm{H}, \mathrm{P}$ \\
\hline Promedio temporal & 27,27 & 11,24 & & 25,54 & 8,03 & G & 28,83 & 11,09 & & 30,84 & 11,80 & $\mathrm{H}$ \\
\hline Promedio nasal & 43,42 & 8,30 & $\mathrm{G}$ & 40,88 & 9,24 & G & 39,43 & 10,37 & & 35,99 & 8,03 & $\mathrm{~N}, \mathrm{H}$ \\
\hline
\end{tabular}

HTO = hipertensos oculares; DE = desviación estándar; $\mathrm{p}=$ diferencias significativas con; NFI = indicador de fibras nerviosas; Desv est TSNIT = desviación estándar TSNIT; Sup/nasal = Cociente superior/nasal; Max mod = Modulación máxima; Ell mod = Modulación de la elipse; Área sup norm = Área superior normalizada; Área inf norm = Área inferior normalizada; Temp//nasal = Cociente Temporal/nasal; Inf/nasal = Cociente inferior/nasal. N: Diferencias con el grupo normal; H: Diferencias con el grupo de HTO; P: Diferencias con el grupo de glaucomas preperimétricos; G: Diferencias con el grupo de glaucomas. 
Tabla III. Áreas bajo la curva ROC e intervalos de confianza de la curva de los parámetros del GDx-VCC para diferenciar entre sanos y sujetos con glaucoma

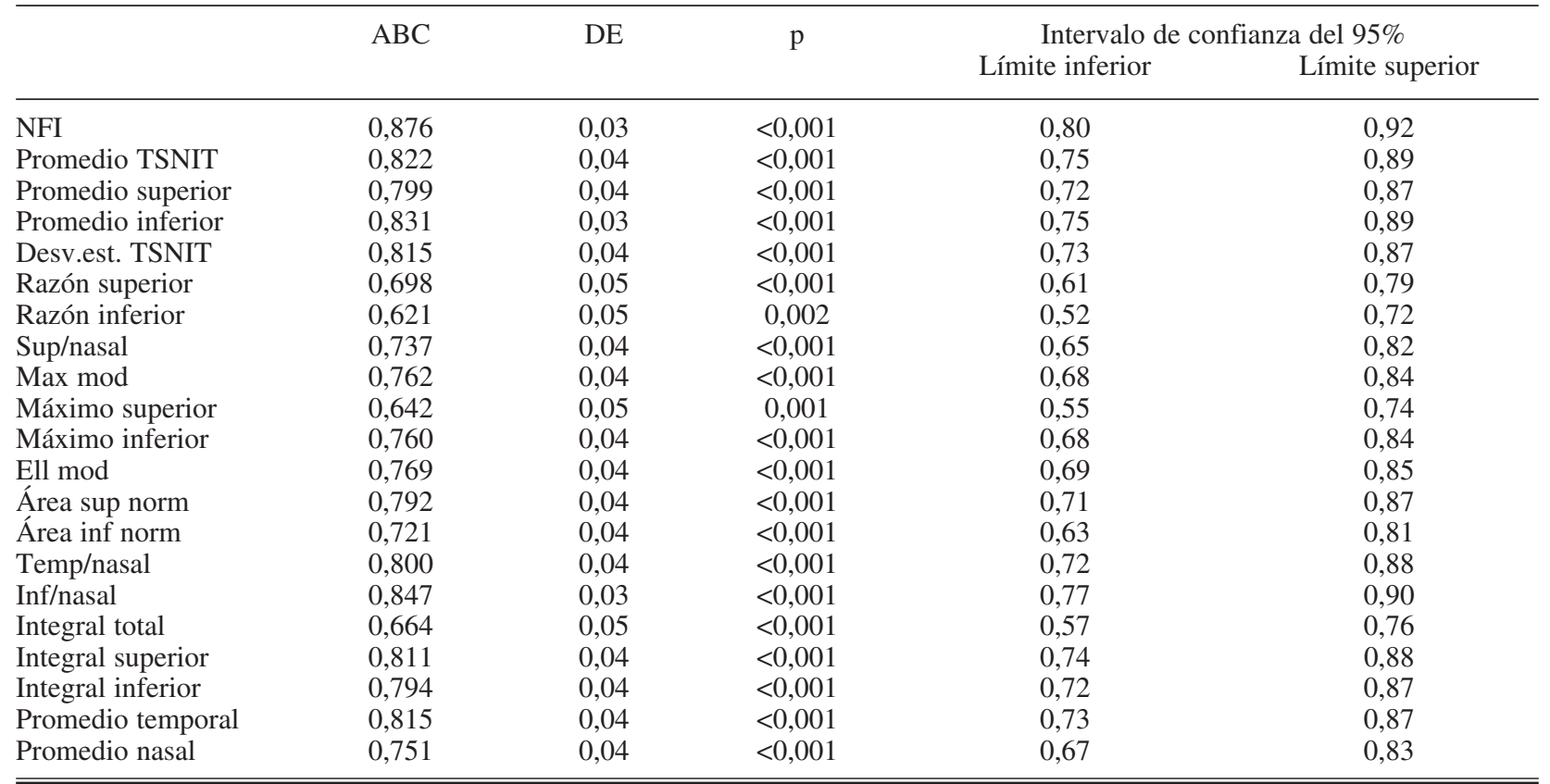

ABC = área bajo la curva ROC; DE = desviación estándar; $\mathrm{p}$ = nivel de significación de la curva ROC; NFI = indicador de fibras nerviosas; Desv est TSNIT = desviación estándar TSNIT; Sup/nasal = Cociente superior/nasal; Max mod = Modulación máxima; Ell mod = Modulación de la elipse; Área sup norm = Área superior normalizada; Área inf norm = Área inferior normalizada; Temp//nasal = Cociente Temporal/nasal; Inf/nasal = Cociente inferior/nasal.

Tabla IV. Sensibilidad de los diferentes parámetros del GDx-VCC para especificidades fijadas en el $85 \%$ y $90 \%$, para discriminar entre sujetos normales y con glaucoma

\begin{tabular}{lrrrr}
\hline & \multicolumn{2}{c}{ Especificidad $85 \%$} & \multicolumn{2}{c}{ Especificidad $90 \%$} \\
& Punto de corte & Sensibilidad $(\%)$ & Punto de corte & Sensibilidad (\%) \\
\hline NFI & 23,50 & 70,42 & 24,50 & 69,01 \\
Promedio TSNIT & 49,99 & 61,97 & 49,58 & 57,74 \\
Promedio superior & 60,73 & 64,78 & 57,41 & 53,52 \\
Promedio inferior & 57,00 & 67,60 & 55,02 & 56,97 \\
Desv.est. TSNIT & 17,11 & 60,56 & 16,84 & 33,80 \\
Razón superior & 1,96 & 47,88 & 1,70 & 33,80 \\
Razón inferior & 2,01 & 52,11 & 1,85 & 22,53 \\
Sup/nasal & 1,72 & 32,39 & 1,61 & 40,84 \\
Max mod & 1,51 & 54,92 & 1,23 & 50,70 \\
Máximo superior & 68,00 & 54,92 & 64,33 & 53,52 \\
Máximo inferior & 70,35 & 60,56 & 66,46 & 66,19 \\
Ell mod & 2,75 & 59,15 & 2,14 & 64,78 \\
Área sup norm & 0,12 & 70,42 & 0,11 & 14,08 \\
Área inf norm & 0,12 & 70,42 & 0,12 & 23,94 \\
Temp/nasal & 1,03 & 32,39 & 1,27 & 56,33 \\
Inf/nasal & 1,79 & 29,57 & 1,69 & 52,11 \\
Integral total & 0,48 & 64,78 & 0,47 & 60,56 \\
Integral superior & 0,19 & 67,60 & 0,18 & 16,90 \\
Integral inferior & 0,18 & 66,19 & 0,18 & 50,70 \\
Promedio temporal & 36,69 & 25,35 & 40,23 & 34,06 \\
Promedio nasal & 34,98 & 52,11 & & \\
\hline \hline
\end{tabular}

NFI = indicador de fibras nerviosas; Desv est TSNIT = desviación estándar TSNIT; Sup/nasal = Cociente superior/nasal; Max mod = Modulación máxima; Ell mod = Modulación de la elipse; Área sup norm = Área superior normalizada; Área inf norm = Área inferior normalizada; Temp//nasal = Cociente Temporal/nasal; Inf/nasal = Cociente inferior/nasal. 
En la tabla IV se encuentran los valores de sensibilidad para especificidades prefijadas al $85 \%$ y $90 \%$ de los parámetros estudiados, para diferenciar entre sanos y glaucomas. El NFI, el área superior normalizada y el área inferior normalizada presentaron las sensibilidades más altas $(70,42 \%$ en los tres parámetros) para una especificidad del $85 \%$. Todos los parámetros del informe estándar mostraron una sensibilidad mayor al $60 \%$ para dicho nivel de especificidad. Para una especificidad del $90 \%$, el NFI sigue siendo el parámetro con mayor sensibilidad, seguido de nuevo por el área superior normalizada y el área inferior normalizada, con unas sensibilidades del $69,01 \%, 66,19 \%$ y $64,78 \%$ respectivamente.

\section{DISCUSIÓN}

En el presente estudio hemos demostrado, al igual que diversos estudios anteriores, que los parámetros analizados por el GDx-VCC son útiles para discriminar entre sujetos normales y glaucomatosos con defectos leves o moderados en la PA $(6,7,17-$ 21). La mayoría de los parámetros presentaron diferencias significativas entre sujetos normales o hipertensos y glaucomatosos, no encontrándose para ningún parámetro diferencias entre controles e hipertensos oculares.

Entre todos los parámetros destaca el NFI como el de mayor capacidad diagnóstica. El NFI es un indicador global del estado de la CFNR, se basa en un avanzado sistema de análisis de redes neuronales que valora el perfil completo de la CFNR y obtiene un número que representa la integridad de la misma. La clasificación diagnóstica del NFI se determinó a partir de la distribución normal de sus valores en la base de datos incluida en el propio GDx VCC.

Otros estudios $(6,7,17)$ han encontrado también que el NFI es el parámetro de mayor rentabilidad diagnóstica para el glaucoma, con ABCs de hasta 0,98 (7). Lógicamente, el grado de la extensión y profundidad de los defectos del campo visual tiene una gran influencia en la sensibilidad de los instrumentos de diagnóstico por imagen. Cuanto más avanzada está la enfermedad mayor sensibilidad presentan estos equipos. Reus y Lemij (7) obtuvieron una capacidad diagnóstica tan elevada para el NFI porque incluyeron una población de glaucomas con más del $50 \%$ de glaucomas severos. Medeiros y col. (6), encontraron resultados más parecidos a los nuestros utilizando una población de glaucomas incipientes (ABC de 0,91 para el NFI). En otra población en nuestro medio (17), los resultados son superponibles, siendo el NFI el parámetro con mayor ABC de nuevo.

$\mathrm{El}$ resto de parámetros que ofrece el GDx-VCC presentaron ABCs menores que el NFI, la mayoría con valores por debajo de 0,80 . Once parámetros de los 21 ofrecidos por el polarímetro presentan sensibilidades superiores al $60 \%$ para una especificidad fijada en el $85 \%$, aunque sólo 5 la siguen manteniendo para una especificidad del $90 \%$. Estos resultados son similares a los de otros estudios como el de DeLeon (21), que presenta 13 parámetros con sensibilidad mayor al $62 \%$ para una especificidad del $80 \%$, a pesar de que en este estudio la población era de predominio afroamericano, que presenta diferencias en la presentación y evolución del glaucoma frente a la raza caucásica.

En nuestro estudio tratamos de valorar la capacidad diagnóstica de los parámetros ofrecidos por el GDx-VCC de manera independiente. Encontramos que cuatro de los cinco parámetros del informe estándar son los que mayor capacidad diagnóstica presentaron de entre todos los que el software del aparato nos ofrece. Es factible que combinando los parámetros en fórmulas discriminantes podamos conseguir una mayor capacidad diagnóstica, como se ha demostrado al evaluar diversos sistemas de evaluación objetiva de la CFNR (20,23-29). En la generación anterior del polarímetro láser, el GDxNFA, mediante la fórmula discriminante propuesta por Weinreb (26), se observó una mayor capacidad diagnóstica que con el «número» que aporta el analizador. Este «número» es el equivalente al NFI del polarímetro láser GDx-VCC.

Hemos de ser cautos a la hora de generalizar los resultados del presente estudio para no sobreestimar los resultados del mismo. Los pacientes provienen de una consulta especializada en glaucoma de un hospital terciario (población seleccionada), por lo que los resultados del estudio en un contexto de consulta oftalmológica primaria serían, con toda seguridad, menores. A pesar de todo esto, encontramos en nuestro estudio una buena capacidad discriminatoria entre controles sanos y sujetos con daño glaucomatoso inicial o moderado mediante el uso del polarímetro láser GDx-VCC. El uso de combinaciones de parámetros u otros métodos de análisis de la CFNR pueden apoyarnos en el diagnóstico precoz de glaucoma y deben ser estudiados. 


\section{BIBLIOGRAFÍA}

1. American Academy of Ophthalmology. Glaucoma Panel. Preferred Practice Pattern. Primary open-angle glaucoma. San Francisco: American Academy of Ophthalmology; 2005. Disponible en: www.aao.org/ppp

2. Quigley HA. Neuronal death in glaucoma. Prog Retin Eye Res 1999; 18: 39-57.

3. Sommer A, Katz J, Quigley HA, Miller NR, Robin AL, Richter RC, et al. Clinical detectable nerve fiber atrophy precedes the onset of glaucomatous field loss. Arch Ophthalmol 1991; 109: 77-83.

4. Weinreb RN, Zangwill L. Retinal nerve fiber layer evaluation in glaucoma. J Glaucoma 2001; 10: S56-S58.

5. Sommer A, Quigley HA, Robin AL, Miller NR, Katz J, Arkell S. Evaluation of nerve fiber layer assessment. Arch Ophthalmol 1984; 102: 1766-1771.

6. Medeiros FA, Zangwill LM, Bowd C, Weinreb RN. Comparison of the GDX VCC scanning laser polarimeter, HRT II confocal scanning ophthalmoscope, and stratus OCT optical coherence tomograph for the detection of glaucoma. Arch Ophthalmol 2004; 122: 827-837.

7. Reus NJ, Lemij HG. Diagnostic accuracy of the GDx VCC for glaucoma. Ophthalmology 2004; 111: 1860-1865.

8. Leung CK, Chan WM, Chong KK, Yung WH, Tang KT, Woo J, et al. Comparative study of retinal nerve fiber layer measurement by StratusOCT and GDx VCC, I: correlation analysis in glaucoma. Invest Ophthalmol Vis Sci 2005; 46: 3214-3220.

9. Weinreb RN, Dreher AW, Coleman A, Quigley H, Shaw B, Reiter K. Histopathologic validation of Fourier-ellipsometry measurements of retinal nerve fiber layer thickness. Arch Ophthalmol 1990; 108: 557-560.

10. Zhou Q, Knighton RW. Light scattering and form birefringence of parallel cylindrical arrays that represent cellular organelles of the retinal nerve fiber layer. Appl Opt 1997; 36: 2273-2285.

11. Bossuyt PM, Reitsma JB, Bruns DE, Gatsonis CA, Glasziou PP, Irwig LM, et al. The STARD statement for reporting studies of diagnostic accuracy: explanation and elaboration. Clin Chem 2003; 49: 7-18.

12. Chylack LT Jr, Wolfe JK, Singer DM, Leske MC; Bullimore MA, Bailey IL, et al. The Lens Opacities Classification System III. The Longitudinal Study of Cataract Study Group. Arch Ophthalmol 1993; 111: 831-836.

13. Tuulonen A, Airaksinen PJ. Initial glaucomatous optic disk and retinal nerve fiber layer abnormalities and their progression. Am J Ophthalmol 1991; 111: 485-490.

14. Caprioli J. Automated perimetry in glaucoma. Am J Ophthalmol 1991; 111: 235-239.

15. Zhou Q, Weinreb RN. Individualized compensation of anterior segment birefringence during scanning laser polarimetry. Invest Ophthalmol Vis Sci 2002; 43: 22212228.

16. Hanley JA, McNeil BJ. A method of comparing the areas under receiver operating characteristic curves derived from the same cases. Radiology 1983; 148: 839-843.
17. Ferreras A, Polo V, Larrosa JM, Pablo LE, Pajarin AB, Pueyo $V$, et al. Can frequency-doubling technology and short-wavelength automated perimetries detect visual field defects before standard automated perimetry in patients with preperimetric glaucoma? J Glaucoma 2007; 16: 372-383.

18. Da Pozzo S, Fuser M, Vattovani O, Di Stefano G, Ravalico G. GDx-VCC performance in discriminating normal from glaucomatous eyes with early visual field loss. Graefes Arch Clin Exp Ophthalmol 2006; 244: 689-695.

19. Reus NJ, Lemij HG. The relationship between standard automated perimetry and GDx VCC measurements. Invest Ophthalmol Vis Sci 2004; 45: 840-845.

20. Lauande-Pimentel $R$, Carvalho RA, Oliveira HC, Gonçalves DC, Silva LM, Costa VP. Discrimination between normal and glaucomatous eyes with visual field and scanning laser polarimetry measurements. Br J Ophthalmol 2001; 85: 586-591.

21. Kanamori A, Nagai-Kusuhara A, Escaño MF, Maeda H, Nakamura M, Negi A. Comparison of confocal scanning laser ophthalmoscopy, scanning laser polarimetry and optical coherence tomography to discriminate ocular hypertension and glaucoma at an early stage. Graefes Arch Clin Exp Ophthalmol 2006; 244: 58-68.

22. Deleón-Ortega JE, Arthur SN, McGwin G Jr, Xie A, Monheit BE, Girkin CA. Discrimination between glaucomatous and nonglaucomatous eyes using quantitative imaging devices and subjective optic nerve head assessment. Invest Ophthalmol Vis Sci 2006; 47: 3374-3380.

23. Bowd C, Chan K, Zangwill LM, Goldbaum MH, Lee TW, Sejnowski TJ, et al. Comparing neural networks and linear discriminant functions for glaucoma detection using confocal scanning ophthalmoscopy of the optic disc. Invest Ophthalmol Vis Sci 2002; 43: 3444-3454.

24. Ford BA, Artes PH, McCormick TA, Nicoela MT, Leblanc $R P$, Chauhan BC. Comparison of data analysis tools for detection of glaucoma with the Heidelberg Retina Tomograph. Ophthalmology 2003; 110: 1145-1150.

25. Weinreb RN, Zangwill LM, Berry CC, Bathija R, Sample $P A$. Detection of glaucoma with scanning laser polarimetry. Arch Ophthalmol 1998; 116: 1583-1590.

26. Medeiros FA, Susanna R Jr. Comparison of algorithms for detection of localised nerve fiber layer defects using scanning laser polarimetry. Br J Ophthalmol 2003; 87: 413419.

27. Essock EA, Zheng Y, Gunvant P. Analysis of GDx-VCC polarimetry data by Wavelet-Fourier analysis across glaucoma stages. Invest Ophthalmol Vis Sci 2005; 46: 2838-2847.

28. Essock EA, Sinai MJ, Bowd C, Zangwill LM, Weinreb RN. Fourier analysis of optical coherence tomography and scanning laser polarimetry retinal nerve fiber layer measurements in the diagnosis of glaucoma. Arch Ophthalmol 2003; 121: 1238-1245.

29. Medeiros FA, Zangwill LM, Bowd C, Bernd AS, Weinreb $R N$. Fourier analysis of scanning laser polarimetry measurements with variable corneal compensation in glaucoma. Invest Ophthalmol Vis Sci 2003; 44: 2606-2612. 